\title{
Comparison of martial artists and healthy individuals using treadmill-induced gait perturbations
}

\author{
Young $\mathrm{DR}^{1,2 *}$, Lee $\mathrm{BC}^{1,2}$ and Layne $\mathrm{CS}^{1,2}$ \\ ${ }^{1}$ Department of Health and Human Performance, University of Houston, Houston, TX, USA \\ ${ }^{2}$ Center for Neuromotor and Biomechanics Research, University of Houston, Houston, TX, USA
}

\begin{abstract}
Falls contribute to $\$ 19$ billion in direct medical costs each year. Physical activity is often recommended to maintain and improve balance into older adulthood. Previous investigations show that several sports, including Tai Chi and martial arts may improve gait and posture. The present study sought to quantify whether martial arts training could improve recovery from gait perturbations such as treadmill induced tripping. Young, healthy martial artists and physically active control participants were recruited for participation. A treadmill tripping was employed to compare martial artists and healthy control participants. Recovery time was the primary outcome and was composed of response step time, the time between perturbation and heel strike of the opposing foot and execution time, the time between response step time and recovery of normal walking. Martial artists exhibited faster recovery times as well as execution times than control participants. Response step time, however, was slower in martial artists. This study suggests that martial arts training, which involves exposure to balance perturbations requiring rapid center of mass adjustments to maintain balance may lead to neuromuscular adaptations and a greater ability to recover from tripping perturbations than physical activities without an inherent balance component.
\end{abstract}

\section{Introduction}

Falls result in $\$ 19$ billion in annual direct medical costs to the American health care system, and are the leading cause of disability in the elderly [1]. Tripping and slipping together account for $59 \%$ of falls, and $57 \%$ of fatal falls [2-3]. Physical activity throughout the lifespan has been recommended to maintain or improve balance into older adulthood. The most frequently suggested physical activities to reduce falls typically involve increased gravitational challenge [4]. Physical activities such as gymnastics, dancing, and Tai Chi require individuals to dynamically move and orient their bodies in space; maintaining balance during rapid changes in center of mass (COM) while executing movements that exceed their limits of stability.

Physical activities that are most commonly recommended for improving balance in young adults tend to involve postural destabilizations that require swift responses to avoid falling. Gymnastics is the most studied in this regard and has been shown to promote postural control development [5]. Previous investigation has suggested that active gymnasts are better able to reweigh and integrate sensory information in order to respond to proprioceptive vibration perturbations and removal of visual feedback with less center of pressure (AP COP) displacement than other athletes [6,7].

A transfer of abilities from specific training to general activities is foundational for the argument that athletic training is useful for fall prevention during aging. Whether skilled individuals can experience a transfer from specific tasks to general tasks has been the subject of scholarly debate for years [8]. Transfer of postural control may be greater in activities that involve unexpected and varied situations where the individual has to adapt to an unpredictable environment [9]. Martial arts forms, including those performed in Karate, Kung Fu, and Taekwondo have been shown to improve cardiorespiratory fitness and balance [10]. These martial arts forms are similar to gymnastics in their predictability, but sparing involves reaction to the opponent and a degree of unpredictability. These martial arts provide consistent excursions of COM outside the limits of stability which require rapid responses to maintain balance. The unpredictability of these circumstances may better approximate environmental challenges such as curbs or ice. Because of these circumstances, martial artist's transfer from training to overall postural control may be improved.

The transfer of skills from specific practice to general application may allow certain athletic populations to recover from being tripped more successfully than others. Maintaining the head in a relatively stable position during gait and when perturbed allows for improved visual feedback as well as improved vestibulo-ocular reflex function [11]. Individuals who are able to maintain upright head posture may be better able to recover from unexpected perturbations. Training in adverse or difficult conditions can lead to strategy that preserves head stability during unexpected gait hazards such as perturbations [12].

This study aimed to identify differences between martial artists and a group of physically active control participants in a treadmill perturbation (i.e. trip) protocol. It was hypothesized that martial arts participants would result in better performance in trip recovery. The results of this investigation may provide evidence to shape recommendations regarding physical activity's role in maintaining balance across the lifespan.

${ }^{\star}$ Correspondence to: Young David Ryan, 4849 Calhoun Road H2 Building Room 2056 Houston, TX, USA, 77204-6015. Tel: 713-743-7677, E-mail: Dyoung6@ uh.edu

Key words: falls, kinematics, locomotion, motion capture, perturbations, recovery

Received: August 28, 2018; Accepted: September 17, 2018; Published: September 20, 2018 


\section{Methods}

\section{Participants}

Participants were recruited from the University of Houston campus as well as targeted through martial arts training centers throughout the Houston metropolitan area. Inclusion criteria were an age of 18-55 years, right foot dominance, no lower body injury over past 12 months and no history of any neurological or other disorder known to negatively impact balance or gait. Exclusion criteria included the inability to stand for ten minutes and the inability to walk for ten minutes. Control participants were recruited if they were physically active in sports or participated in exercising that had no specific balance training components for a minimum of three hours per week [13]. Examples of disqualifying activities included gymnastics and ballet. Martial arts participants were recruited if they had practiced any active martial art for a minimum of five years for three hours per week.

Prior to data collection, participants provided informed consent through a process in accordance with the Helsinki Declaration approved by the University of Houston's institutional review board for experimental studies. Participants also completed a PAR-Q document to ensure they were healthy enough to exercise. The PAR-Q is a widely used tool to screen candidates prior to exercise [14]. Participants completed a physical activity history questionnaire which included their primary mode of exercise, training years, and hours per week trained as well as demographic information, including age, height $(\mathrm{cm})$, bodyweight $(\mathrm{kg})$, and leg length $(\mathrm{cm})$ were recorded.

\section{Experimental procedure}

Participants were exposed to simulated 'trips' during treadmill gait. Anthropometric data were recorded prior to applying 39 reflective markers on bony landmarks across the arms, legs, trunk and head using the Plug-In-Gait virtual kinematic model of Vicon Nexus (Vicon; Oxford Metrics Ltd, Oxford, England). Participants were then fitted with a harness which was securely fastened to steel beams above the treadmill to eliminate falling after a failed trip recovery. Static participant calibration and establishment of a preferred gait speed were then performed prior to the data collection. Preferred gait speed was identified by gradually increasing treadmill speed until the participant vocalized that their preferred gait speed had been reached. Following this, participants were instructed to maintain that speed for two minutes to familiarize themselves with walking on a split belt treadmill. Participants walked on a split belt treadmill with a force plate embedded under each belt (Bertec Co., Columbus, $\mathrm{OH}$ ).

The protocol consisted of 10 walking trials, five of which included a simulated trip. The first simulated trip was used as a familiarization trial, and was excluded from analysis. Trips were produced by a temporary stoppage of the left treadmill belt. This stoppage was triggered when the participant's ground reaction force (GRF) surpassed a threshold of $10 \%$ of their bodyweight on the indicated stride [15]. The left belt returned to previous velocity within $100 \mathrm{~ms}$ after the participant made their first contact with the right treadmill belt [16]. Contact was identified using the first measurable GRF placed on the right force plate. Trips always occurred on the left foot and were administered on a random stride between strides 10 and 20 of each trial. Custom software was utilized in production of perturbation and collection of data synchronized with perturbation time. This software was described previously by Lee [17]. In between each trial participants were provided a 30 second break.

\section{Data processing}

Marker positions were collected at a sampling rate of $100 \mathrm{~Hz}$ from a 12-camera motion capture setup and were processed using Vicon's Plug-in-Gait model to obtain joint kinematics (Vicon, Centennial, $\mathrm{CO})$. Marker positions were used to calculate maximum head flexion velocity as a barometer of stability of vestibular and visual feedback. Marker positions were also used to calculate maximum center of mass (COM) velocity during the perturbation. Ground reaction force (GRF) data from the treadmill's embedded force plates were obtained at a sampling frequency of $1000 \mathrm{~Hz}$ and exported. Ground reaction force data were filtered using a low-pass second-order Butterworth filter with a $10 \mathrm{~Hz}$ cut-off frequency [16]. Custom MATLAB codes were used to calculate peak vertical GRF force normalized to participant bodyweight, recovery, response, and execution time from the GRF data (The MathWorks, Natick, MA).

Recovery Time (RT) was defined as the total time between the perturbation and re-establishment of normal gait, as computed using the GRF profile $[16,17]$. RT was divided into two components. The first component was Response Step Time (RST), which was defined as the time lapse between perturbation incident and first heel strike of the following foot, and was also computed automatically using the GRF profile (Figure 1). The second component of RT was Execution time (ET), which was defined as the difference between RT and RST and constituted the final component of the recovery.

\section{Statistical analysis}

Between group differences were identified using SPSS statistical software (SPSS Inc, Chicago, Illinois, USA). To test for differences in demographic information, two-tailed Student's t-tests were employed. Assumptions of equal variance between groups and normalcy were tested. The test was unable to reject the null hypothesis of equal variance and normalcy. A comparison of between groups recovery performance was conducted by employing one-tailed Student's t-tests comparing the groups' recovery time, response step time, and execution time. Similar measures were performed comparing peak center of mass velocity, peak trunk flexion velocity, and peak head angle velocity. One-tailed tests were chosen based on the foundation of literature demonstrating that martial artists typically outperform others in dynamic balance tasks. When significant results were identified, effect sizes ( $\eta \mathrm{p} 2)$ and 95\% confidence intervals (CI) are also presented. Hedge's G effect size calculations were employed due to a sample size of ten control participants and nine martial artists. For the same reason, a t-statistic using sample means and standard deviations was used to compute confidence intervals. For all statistical analyses, significance was set at $(\mathrm{p}<0.05)$.

\section{Results}

\section{Participant Characteristics}

Groups did not differ significantly in age, height, body mass or leg length. Participants also reported a similar number of years training and hours spent training per week. Preferred walking speed and speed normalized to their leg length were not significantly different between the two groups (Table 1).

\section{Experimental protocol}

Martial artists recovered significantly faster than the control group $(\mathrm{p}=0.032$; Large effect). Execution Time (ET) was lower in martial artists than control participants ( $\mathrm{p}=0.018$; Large effect). Conversely, 


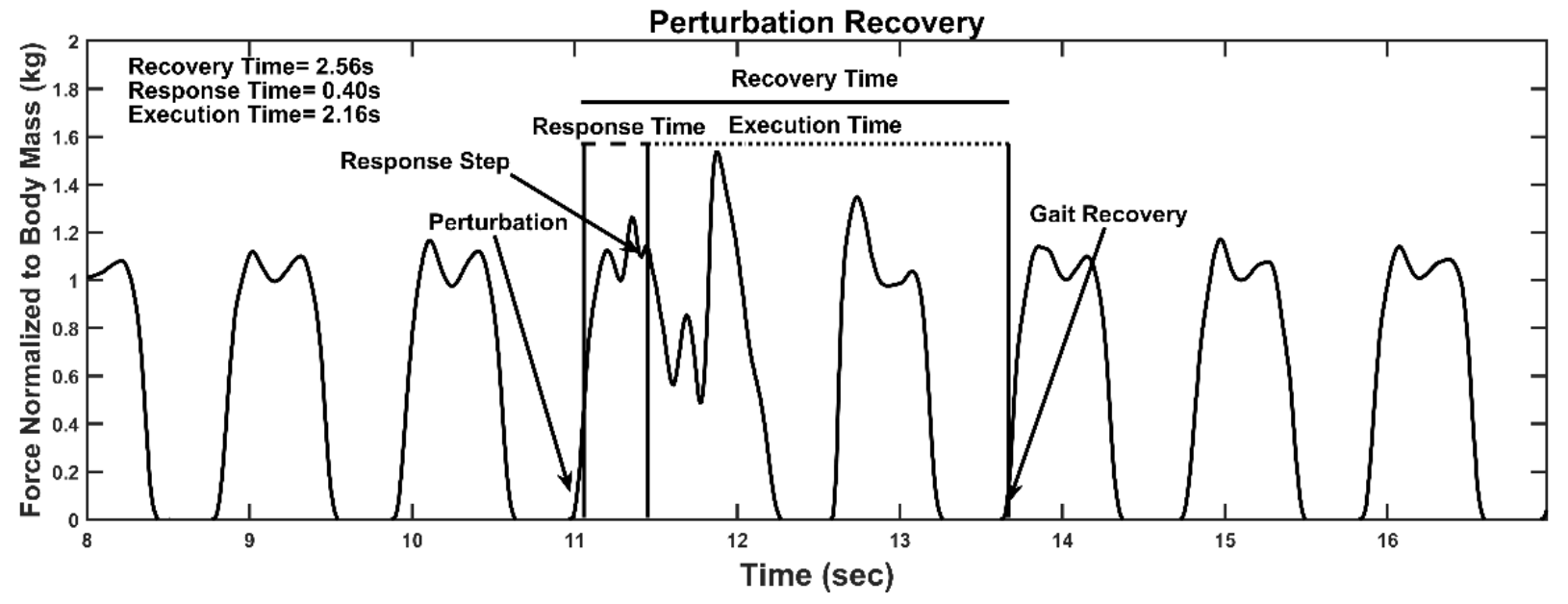

Figure 1. Example of temporal characteristics of perturbation recovery. P represents perturbation time. ET is the time span between RST and RT

Table 1. Demographic Data. Statistical analysis performed using two-tailed Student's t-tests

\begin{tabular}{|l|c|c|c|}
\hline Participant Demographics & Martial Arts & Control & p \\
\hline N $(\mathrm{sex})$ & $9(7 \mathrm{~m})$ & $10(6 \mathrm{~m})$ & 0.29 \\
\hline Age & $28.22 \pm 8.71$ & $24.7 \pm 4.99$ & 0.34 \\
\hline Training Years & $14.44 \pm 8.82$ & $6.58 \pm 8.45$ & 0.06 \\
\hline Training Hours & $10.72 \pm 11.33$ & $14 \pm 6.8$ & 0.32 \\
\hline Height $(\mathrm{cm})$ & $176.33 \pm 9.52$ & $168.8 \pm 10.78$ & 0.13 \\
\hline Weight $(\mathrm{kg})$ & $81.14 \pm 21.21$ & $74.65 \pm 17.14$ & 0.47 \\
\hline Leg Length $(\mathrm{cm})$ & $92.83 \pm 5.15$ & $91.9 \pm 5.95$ & 0.72 \\
\hline Walking Speed $(\mathrm{m} / \mathrm{s})$ & $0.88 \pm 0.17$ & $0.91 \pm .14$ & 0.66 \\
\hline Normalized Speed $\mathrm{m} / \mathrm{s} / \mathrm{cm}$ & $0.0095 \pm 0.002$ & $0.0099 \pm 0.0014$ & 0.62 \\
\hline
\end{tabular}

average Response Step Time (RST), was significantly lower in control participants $(\mathrm{p}=0.028$, Small effect). Confidence intervals and $\eta \mathrm{p} 2$ scores are included (Table 2). Comparisons of kinematic outcomes between groups revealed no differences in maximum trunk flexion, CoM velocity or average peak normalized GRF between martial artists and control participants. However, maximum head flexion velocity was higher in control participants than martial artists $(\mathrm{p}<0.0001$; $\eta p 2=6.03$ Large effect).

\section{Discussion}

The current investigation is the first to compare the recovery during a tripping perturbation task between martial artists and healthy, active individuals. While Gatts, et al. (2007) found that Tai Chi training improved recovery properties in response to a trip in adults aged 68-92, no trip investigation has studied active martial arts (e.g. Karate, Kung $\mathrm{Fu}$, Taekwondo) focusing on younger individuals [18]. The current investigation found that experienced martial artists took longer to perform their initial response to the trip but executed their recovery strategy more quickly than the control participants, which may be a result of their training. As mentioned, martial arts focus of making rapid, forceful movements that often bring their COM momentarily outside of the postural base of support. To effectively maintain balance, martial artists must learn to rapidly adapt their body configuration so that they reestablish their base of support prior to losing their balance. Their training regime therefore provides them with extensive experience recovering from momentary excursion beyond their base of support. It is likely that this experience has provided them confidence in their ability to rapidly and accurately assess the consequences of a trip and therefore delay their initial response in favor of developing and executing an effective recovery from a trip. Our findings support the above explanation. In contrast, the control group, who were physically active but did not regularly experience systematic and repeated segmental body configurations that would bring their COM outside their base of support, would not be expected to develop recovery strategies that include delaying their initial response to a trip. Additionally, it is reasonable to speculate that because of their relative lack of experience, the control group would also demonstrate less efficient execution of their recovery strategies. Head stability was greater in the martial arts group has been reported to be important for optimal functioning of the visual and vestibular system, both of which are important for postural control $[12,17,19]$.

In this investigation, Recovery Time was comprised of two components: Response Step Time, and Execution Time. RST was faster in the control group than the martial arts group. Previous research into the recovery of walking perturbations has suggested that the initial response is rapidly initiated and mediated by spinal mechanisms producing a stereotypical pattern of lower limb muscle activity [20,21]. The timing of the initiation of 'automatic' responses to induced tripping can be dissociated from the motor commands associated with the response depending upon both the available sensory feedback and experience [16]. In the current protocol, the martial artists were able to delay their initial 'automatic' response relative to the controls, a finding we consider consistent with the type of training martial artists participate in. Although the initial response phase is spinally-mediated, the execution phase is subject to increased cortical influence and therefore is more amenable to the effects of training [22,19].

The results support previous findings that specific types of training can transfer to more general types of activities. In this case, training composed of vigorous motions that result in exceeding the postural base of support appear to have effectively transferred to the ability to efficiently recover from an induced trip. While the control group was physically active, their more generalized training did not include regular exposure to unexpected destabilizations unlike the martial arts group who, through sparing, experience them regularly. Although the control participants were able to effectively recover from the induced trips, their longer execution times and decreased head stability suggest a less effective response pattern than that exhibited by the martial artists. The findings provided support to this investigation's hypothesis that martial artists would outperform the control group in trip recovery. Important differences were discovered in recovery time, execution time 
Table 2. Recovery Characteristics. Numbers expressed as mean \pm SD alongside 95\% confidence intervals and effect sizes. Statistical analysis performed using one-tailed Student's t-tests. Statistical significance expressed by *

\begin{tabular}{|l|c|c|c|c|}
\hline Recovery Characteristics & \multicolumn{1}{|c|}{ MA } & Control & p & ES $\mathbf{~}$ \\
\hline Recovery time (s) & $2.14 \pm 0.47 \mathrm{~s}[1.78-2.50]$ & $2.51 \pm 0.35 \mathrm{~s}[2.26-2.76]$ & $0.03^{*}$ & 0.9 \\
\hline Response step time (s) & $0.44 \pm 0.05 \mathrm{~s}[0.40-0.48]$ & $0.37 \pm 0.09 \mathrm{~s}[0.31-0.43]$ & $0.01^{*}$ & 0.95 \\
\hline Execution time (s) & $1.8 \pm 0.54 \mathrm{~s}[1.39-2.22]$ & $2.18 \pm 0.39 \mathrm{~s}[1.90-2.46]$ & $0.047^{*}$ & Large \\
\hline Max head angle velocity (DPS) & $5.97 \pm 0.36[5.70-6.25]$ & $8.02 \pm 3.2[7.79-8.25]$ & $<0.001^{*}$ & 0.81 \\
\hline
\end{tabular}

as well as their ability to maintain head stability, thereby providing for effective visual and vestibular sensory integration.

Our results indicate that martial artists exhibit a different overall response pattern that can be considered more efficient than physically active controls during an induced trip. These results suggest that martial artists training regime that often results in unexpected movements bringing their center of mass outside their base of support, and the associated rapid corrective responses transferred to their recovery from the induced trips. The above findings provide evidence that martial arts may reduce chances of falling after being tripped and strengthen the argument that martial arts should be considered as a potential training tool throughout youth and adulthood to improve balance and provide a protective effect against falls as one ages. Additional investigation is required to identify underlying mechanisms associated with trip recovery to gain a greater understanding regarding why martial artists outperform healthy, active individuals and if the benefits martial arts training can be transferred to additional activities requiring precisely controlled posture.

\section{Authors' contributions}

DRY and CSL conceived the study idea. DRY, CSL and BCL devised the study methodology, data processing and analysis. DRY was primarily responsible for manuscript preparation. DRY, CSL and BCL provided comments and agreed on the final version of the manuscript.

\section{Conflict of interest statement}

All authors report that there are no financial conflict of interests or financial or personal relationships with people or organizations that could inappropriately influence this work.

\section{Acknowledgements}

We would like to acknowledge the work of our undergraduate assistants and the volunteers who participated in the study.

\section{References}

1. Stevens JA, Corso PS, Finkelstein EA, Miller TR (2006) The costs of fatal and nonfatal falls among older adults. Inj Prev 12: 290-295. [Crossref]

2. Berg WP, Alessio HM, Mills EM, Chen T (1997) Circumstances and consequences of falls in independent community-dwelling older adults. Age Ageing 26: 261.

3. Shiller JS, Kramarow EA, Dey AN (2007) Fall injury episode among noninstitutionalized older adults: United States, 2001-2003. Adv Data 21: 1-16.
4. Arkov VV, Abramova TF, Nikitina TM, Ivanov VV, Suprun DV, et al. (2009) Comparative study of stabilometric parameters in sportsmen of various disciplines. Bull Exp Biol Med 147: 233-235.

5. Garcia C, Barela JA, Viana AR, Barela AMF (2011) Influence of gymnastics training on the development of postural control. Neurosci Lett 492: 29-32.

6. Vuillerme N, Danion F, Martin L, Boyadjian A, Prieur JM, et al. (2001) The effect of expertise in gymnastics on postural control. Neurosci Lett 303: 83-86.

7. Vuillerme N, Teasdale N, Nougier V (2001). The effect of expertise in gymnastics on proprioceptive sensory integration in human subjects. Neurosci Lett 311: 73-76.

8. Barnett SM, Ceci SJ (2002) When and where do we apply what we learn? A taxonomy for far transfer. Psychol Bull 128: 612-637. [Crossref]

9. Perrot C, Mur JM, Mainard D, Barrault D, Perrin PP (2000) Influence of trauma induced by judo practice on postural control. Scand J Med Sci Sports 10: 292-297.

10. Origua Rios S, Marks J, Estevan I, Barnett LM (2018) Health benefits of hard martial arts in adults: a systematic review. J Sports Sci 36: 1614-1622. [Crossref]

11. Cromwell RL, Newton RA, Carlton LG (2001) Horizontal plane head stabilization during locomotor tasks. J Mot Behav 33: 49-58.

12. Bucello-Stout RR, Cromwell RL, Bloomberg, JJ, Whorton EB (2013) Sensorimotor adaptation training's effect on head stabilization in response to a lateral perturbation in older adults. J Aging Phys Act 2013: 272-289.

13. Haskwell WL, Pate RR, Powell KE, Blair SN, Franklin BA, et al. (2007) Physical activity and public health: Updated recommendation for adults from the american college of sports medicine and the american heart association. Med Sci Sports Exerc 39: 1423-1434.

14. Thomas S, Reading J, Shephard RJ (1992) Revision of the physical activity readiness questionnaire (PAR-Q). Can J Sport Sci 17: 338-345.

15. Perry J, Burfield J (2010) Gait analysis: Normal and pathological function. Second Edition. Thorofare (New Jersey): SLACK Incorporated. pp:576

16. Lee BC, Martin BJ, Thrasher TA, Layne CS (2017) The effect of vibrotactile cuing on recovery strategies from a treadmill-induced trip. IEEE Trans Neural Syst Rehabil Eng 25: 235-243. Available from: https://doi.org/10.1109/TNSRE.2016.2556690

17. Lee BC, Thrasher TA, Layne CS, Martin BJ (2016) Vibrotactile cuing revisited to reveal a possible challenge to sensorimotor adaptation. Exp Brain Res 234: 1-8.

18. Gatts SK, Woollacott MH (2007) How Tai Chi improves balance: biomechanics of recovery to a walking slip in impaired seniors. Gait Posture 25: 205-214. [Crossref]

19. Dijkstra BW, Horak FB, Kamsma YPT, Peterson DS (2015) Older adults can improve compensatory stepping with repeated postural perturbations. Front Aging Neurosci 7: 1-8.

20. Dietz V, Quintern J, Berger W (1984) Corrective reactions to stumbling in man: Functional significance of spinal and transcortical reflexes. Neurosci Lett 44: 131-135.

21. Dietz V, Quintern J, Berger W (1986) Stumbling reactions in man: Release of a ballistic movement pattern. Brain Res 362: 355-357.

22. Bieryla KA, Madigan ML, Nussbaum MA (2007) Practicing recovery from a simulated trip improves recovery kinematics after an actual trip. Gait Posture 26: 208-213.

Copyright: (C2018 Young DR. This is an open-access article distributed under the terms of the Creative Commons Attribution License, which permits unrestricted use, distribution, and reproduction in any medium, provided the original author and source are credited. 